\title{
Takayasu arteritis presenting with spontaneous pneumothorax
}

\author{
Mina Hizal $^{1 \oplus}$, Selcan Demir ${ }^{2 \oplus}$, Sanem Eryılmaz Polat ${ }^{1 \oplus}$, Seza Özen ${ }^{2 \oplus}$, Nural Kiper ${ }^{1 \oplus}$
}

Divisions of ${ }^{1}$ Pediatric Pulmonology and ${ }^{2}$ Pediatric Rheumatology, Department of Pediatrics, Hacettepe University Faculty of Medicine, Ankara, Turkey.

\begin{abstract}
Background. Takayasu arteritis (TA) is an idiopathic chronic inflammatory arteritis that affects the large blood vessels. Pulmonary involvement was considered an uncommon manifestation of the disease and spontaneous pneumothorax has not been previously described in association with TA.

Case. We report a 13-year-old female who had TA complicated by spontaneous pneumothorax during treatment. She was admitted to the hospital reporting difficulty standing from a squatting position and inability to walk without support. She had been diagnosed with dilate cardiomyopathy four years ago and cardiac functions had deteriorated over time. Catheter angiography revealed diffuse narrowing of the abdominal aorta. In magnetic resonance angiography, total-subtotal occlusion of the infrarenal abdominal aorta in a $2 \mathrm{~cm}$ area and subtotal occlusion of the left renal artery were detected without pulmonary artery involvement. Methotrexate, azathioprine, and prednisolone were administered. Tension pneumothorax developed on the left side while she was on prednisolone treatment.
\end{abstract}

Conclusion. To our knowledge, this is the first case of spontaneous pneumothorax associated with TA to be reported in the literature.

Key words: spontaneous pneumothorax, Takayasu arteritis.

Takayasu arteritis (TA) is an idiopathic chronic inflammatory arteritis that affects the large blood vessels, predominantly the aorta, its major branches, and the pulmonary artery. ${ }^{1,2}$ The clinical signs and symptoms of TA are quite variable and depend on the localization, and degree and severity of vascular involvement. ${ }^{3}$ The pulmonary manifestations of TA include cough, dyspnea, chest pain, and pulmonary hypertension. ${ }^{4}$ In the past, because of the rare presentation of pulmonary symptoms, pulmonary involvement was considered an uncommon manifestation of the disease; however, the prevalence of pulmonary arterial involvement is now estimated to be between $50 \%$ and $80 \% .^{5}$

$凶 \quad$ Mina Hizal

minahizal@outlook.com

Received 17th November 2019, revised 27th January 2020, accepted 18th February 2020.
Pneumothorax is defined as the presence of air in the pleural space. ${ }^{6}$ Spontaneous pneumothorax is a type of pneumothorax that occurs without trauma. ${ }^{7}$ Pneumothorax is classified as both primary and secondary; primary pneumothorax occurs without underlying lung disease and secondary pneumothorax occurs in patients with underlying pulmonary disease. ${ }^{8}$

Spontaneous pneumothorax can be observed in various types of lung disease; however, rheumatologic diseases such as systemic lupus erythematosus (SLE), rheumatoid arthritis, antineutrophil antibody (ANCA) with associated vasculitis, scleroderma, and dermatomyositis are rarely associated with pneumothorax. ${ }^{9}$ The majority of the information reviewed for this research was obtained from a series of case studies and reports. To our knowledge, spontaneous pneumothorax has not been 
described in association with TA in previous literature.

\section{Case Report}

This case was of a 13-year-old female who was admitted to the hospital reporting difficulty standing from a squatting position as well as the inability to walk without support, which she had had for at least two months. Symptoms of vomiting, nausea, and loss of appetite were also reported. At the age of nine years, the patient had been diagnosed with dilated cardiomyopathy and was treated with diuretics, angiotensinconverting enzyme inhibitors, and digoxin. For the following 4 years, as the patient's cardiac function worsened, catheter angiography was performed, which revealed diffuse narrowing of the abdominal aorta. Acute- phase reactants were high and oral steroid $(1 \mathrm{mg} / \mathrm{kg} /$ day $)$ and azathioprine (100 mg/day) were started because of a probable diagnosis of TA. One month later, acute-phase reactants were still high and azathioprine treatment was changed to methotrexate (15 mg/ week). After three weeks, she developed pancytopenia and received three pulses of intravenous methylprednisolone (500 mg). Subsequently, the patient was referred to our hospital for further evaluation. On admission, her height and weight were $140 \mathrm{~cm}$ and $28 \mathrm{~kg}$, respectively. On physical examination, she was irritable, her body temperature was $39^{\circ} \mathrm{C}$, heart rate was 100 beats per minute, blood pressure was within normal range in the four extremities, lower-limb pulses were weakly palpable, upper-limb pulses were present and equal. A 3/6 systolic murmur was audible over the cardiac apex. Her muscle strength was grade $4 / 5$ in the upper limbs and grade $3 / 5$ in the lower limbs. The remaining physical examination was unremarkable.

Her laboratory findings showed leukopenia

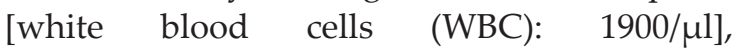
anemia [hemoglobin ( $\mathrm{Hb}): 10.6 \mathrm{~g} / \mathrm{dl}$ ], and thrombocytopenia (platelets: 51,000/ $\mu \mathrm{l}$ ). Inflammatory biomarkers such as C-reactive protein and the erythrocyte sedimentation rate (ESR) were both elevated [44.9 (normal: 0-0.8) $\mathrm{mg} / \mathrm{dl}$ and 60 (normal: 0-20) $\mathrm{mm} / \mathrm{h}$, respectively]. Blood glucose, liver and renal function parameters, and muscle enzymes were all within the normal range. The tests for anti-neutrophil cytoplasmic antibodies (ANCA), anti-nuclear antibody, anti-double stranded DNA (anti-dsDNA), and rheumatoid factor were negative. Complement levels were normal. Echocardiography showed severe generalized left ventricles dysfunction with an ejection fraction of $25 \%$.

Magnetic resonance (MR) angiography revealed total-subtotal occlusion of the infrarenal abdominal aorta in a $2 \mathrm{~cm}$ area, and subtotal occlusion of the left renal artery. Pulmonary arterial involvement was not detected and the diagnosis of Takayasu arteritis was confirmed. Methotrexate treatment was stopped because of pancytopenia and prednisolone was continued at a dosage $1 \mathrm{mg} / \mathrm{kg} /$ day. Nontyphoidal salmonella group D was identified through a blood culture and amikacin and ciprofloxacin were started. The muscle weakness was thought to be due to steroid myopathy. She was not a candidate for anti-tumor necrosis factor (TNF) or antiinterleukin (IL)-6 agents as immunosuppressive therapy owing to her impaired cardiac function and thrombocytopenia. During her follow-up on antibiotic and steroid treatment, the patient was evaluated for lung involvement because she required oxygen supplementation.

Respiratory osculation and the chest radiography findings were normal. A pulmonary function test revealed forced expiratory flow in one second $\left(\mathrm{FEV}_{1}\right) 1.62$ liters (70\% predicted), forced vital capacity (FVC) 1.36 liters (68\% predicted) and $\mathrm{FEV}_{1} / \mathrm{FVC} 83 \%$. As a result, extensive diagnostic tests were planned and the examination of the patient was remarkable for the absence of breath sounds in the left lung. Chest X-ray revealed leftsided pneumothorax (Fig. 1). Chest computed tomography (CT) was performed to evaluate the pulmonary parenchyma, which showed tension pneumothorax on the left side of the lung (Fig. 


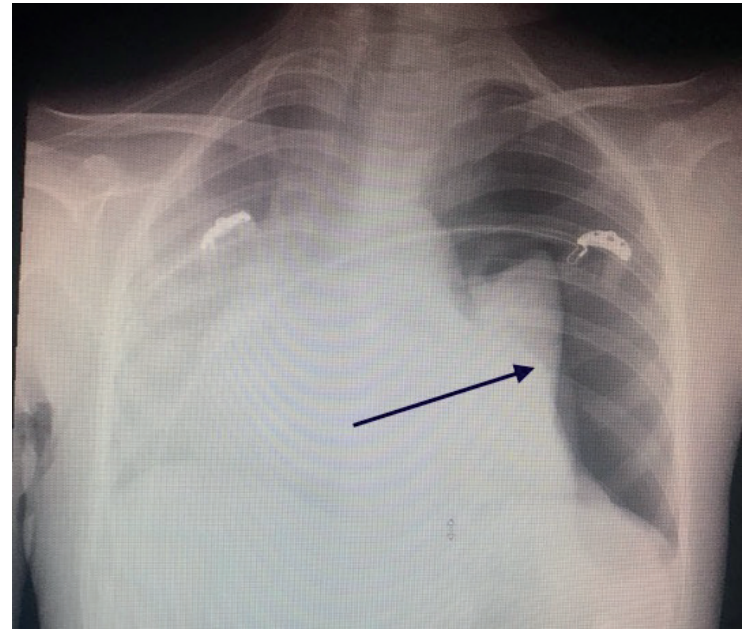

Fig. 1. Posteroanterior chest X-ray showing pneumothorax on the left site.

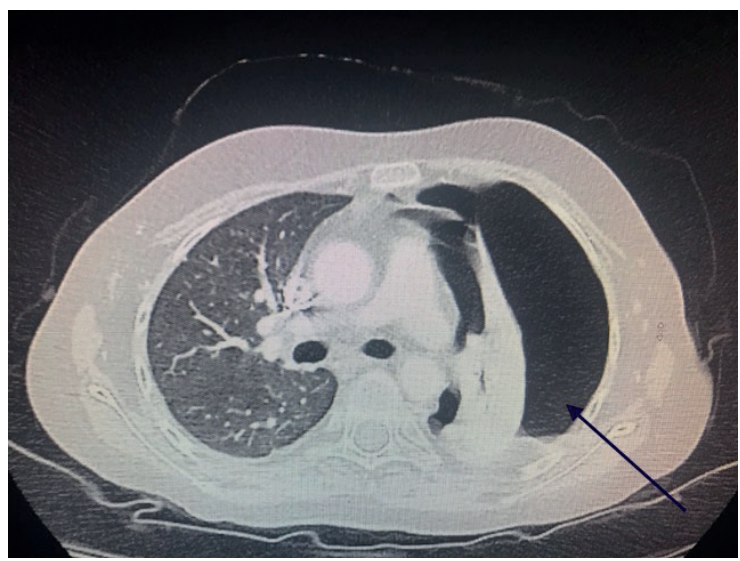

Fig. 2. Pulmonary CT scan reveals tension pneumothorax on the left side.

2). A chest tube was placed and the lung was re-expanded while the pleural cultures were negative. The chest tube was then removed after five days. Extensive viral tests were also negative.

After 14 days of antibiotic therapy, the patient's cytopenia improved and tocilizumab $(10 \mathrm{mg} / \mathrm{kg})$ treatment was started and as a result the steroid treatment dose was reduced. After 3 months on tocilizumab treatment, the patient died of heart failure.

Informed consent was received from the families for publication of the case.

\section{Discussion}

Even though pulmonary involvement is seen in more than half of all patients with TA, predominant pulmonary involvement and its symptoms are not common. Most of the pulmonary symptoms mentioned in the literature were due to the pulmonary arterial involvement of the disease. Pulmonary parenchymal involvement is also rarely seen in TA; however, in the literature, there are reports of unusual manifestations of pulmonary involvement. ${ }^{10} \mathrm{~A}$ case of TA with severe respiratory failure associated with bilateral parenchymal infiltrations that responded well to steroid treatment was described by Cilli et al. $^{11}$

Takahashi et al. ${ }^{2}$ evaluated the CT findings of the pulmonary parenchyma in 25 patients with TA and found low attenuation areas in the lungs of 11 patients, subpleural reticulolinear changes in 12 patients, and pleural thickening in nine patients. Their results suggested that regional hypoperfusion was responsible for the appearance of low attenuation pulmonary areas. They also made speculations regarding pulmonary thromboembolism in other CT findings for the pleura and adjacent lung.

The interesting feature of this case is the development of spontaneous pneumothorax, which is rarely associated with systemic inflammatory disease. ${ }^{9}$ According to different studies, the incidence of pneumothorax in Wegener's granulomatosis (WG) is between 3 to $5 \% .^{12,13}$ In certain reported cases of WG, the occurrence of pneumothorax was attributed to the rupture of cavitary lesions and immunosuppressive treatment. ${ }^{9}$

In a literature review by Tanaka et al., ${ }^{13} 11$ patients with recurrent pneumothorax with SLE were evaluated. All patients had underlying pulmonary involvement such as interstitial lung disease, pulmonary suppuration, pulmonary hemorrhage, and pulmonary embolism or cyst formation. Additionally, all but one of the 11 patients in this study used glucocorticoid treatment. 
Glucocorticoids have been shown to cause tissue fragility by creating adverse effects on both growth factors, as well as collagen deposition during wound healing. ${ }^{13}$ Tissue fragility may be among the factors contributing to pneumothorax. On the other hand, the occurrence of pneumothorax has only been reported in a few cases, and the use of methotrexate and azathioprine, and their potential contribution to pneumothorax is unclear. $^{14}$ There is no data in the literature that suggest that methotrexate-induced parenchymal lung disease can predispose to the development of pneumothorax and bronchopleural fistula. ${ }^{15}$ Gotsman et al. ${ }^{16}$ described a case of pneumothorax secondary to the cavitation of a rheumatoid nodule during methotrexate treatment. It was speculated that methotrexate might have aggravated the previous pulmonary involvement. Also, there are no case reports indicating an association of azathioprine with pneumothorax. This association should be investigated further because it remains unclear.

Acute bacterial pneumonias caused by Staphylococcus aureus, Klebsiella pneumoniae, Pseudomonas aeruginosa, Streptococcus pneumoniae, have all been associated with pneumothorax. Also, infectious agents such as Pneumocystis jiroveci pneumonia, fungal pneumonia and tuberculosis have also been associated with pneumothorax. ${ }^{17}$ In these situations, pulmonary cavity formation due to infections are risk factors for the development of pneumothorax. ${ }^{18}$ In our patient, there was no evidence of viral or bacterial pneumonia, but she had salmonella bacteremia, which has not previously been shown associated with pneumothorax development.

The underlying pathologic mechanism of pneumothoraxinourpatienthasnotyetbeenfully determined. We think that many factors such as glucocorticoids use, other immunosuppressive treatments, and the pulmonary involvement of TA may have contributed to the occurrence of pneumothorax. As a result, close monitoring of patients is important because pneumothorax, which occurs in rheumatic diseases, tends to be recurrent. ${ }^{13}$

To our knowledge, this is the first description of a patient with spontaneous pneumothorax associated with TA. Although there are other factors that may contribute to the development of pneumothorax, this finding can be considered as part of the wide spectrum of TA.

\section{REFERENCES}

1. Moll R, Hameed B. A case of Takayasu arteritis diagnosed at early-phase of disease by conventional CT scanning. Int J Rheum Dis 2012;15: e63-e64.

2. Takahashi K, Honda M, Furuse M, Yanagisawa M, Saitoh K. CT findings of pulmonary parenchyma in Takayasu arteritis. J Comput Assist Tomogr 1996; 20: 742-748.

3. Toledano K, Guralnik L, Lorber A, et al. Pulmonary arteries involvement in Takayasu's arteritis: two cases and literature review. Semin Arthritis Rheum 2011; 41: 461-470.

4. Nakajima N. Takayasu arteritis: consideration of pulmonary involvement. Ann Vasc Dis 2008; 1: 7-10.

5. Arestis NJ, MacNee W, Wightman AJA, Murchison JT. Takayasu arteritis with predominantly pulmonary involvement diagnosed by CT angiography. Respiratory Medicine CME 2008; 1: 278-280.

6. Noppen M. Spontaneous pneumothorax: epidemiology, pathophysiology and cause. Eur Respir Rev 2010; 19: 217-219.

7. Onuki T, Ueda S, Yamaoka M, et al. Primary and secondary spontaneous pneumothorax: prevalence, clinical features, and in-hospital mortality. Can Respir J 2017; 2017: 6014967.

8. Luh SP. Review: Diagnosis and treatment of primary spontaneous pneumothorax. J Zhejiang Univ Sci B 2010; 11: 735-744.

9. Ouellette DR, Parrish S, Browning RF, et al. Unusual causes of pneumothorax. J Thorac Dis 2014; 6(Suppl 4): S392-S403.

10. Yang CD, Teng JL, Gu YY, Chen SL. Takayasu's arteritis presenting with bilateral pulmonary granulomatosis. Clin Rheumatol 2007; 26: 612-614.

11. Cilli A, Ozdemir T, Ogus C. Takayasu's arteritis presenting with bilateral parenchymal consolidations and severe respiratory failure. Respiration 2001; 68: 628-630. 
12. Belhassen-Garcia M, Velasco-Tirado V, AlvelaSuarez L, et al. Spontaneous pneumothorax in Wegener's granulomatosis: case report and literature review. Semin Arthritis Rheum 2011; 41: 455-460.

13. Tanaka N, Kusunoki Y, Kaneko K, et al. Systemic lupus erythematosus complicated by recurrent pneumothorax: case report and literature review. Nihon Rinsho Meneki Gakkai Kaishi 2010; 33: 162168.

14. Hauben M, Hung EY. Pneumothorax as an adverse drug event: an explor- atory aggregate analysis of the US FDA AERS database including a confounding by indication analysis inspired by Cornfield's condition. Int J Med Sci 2013; 10: 965-973.

15. Taha A, Hazam R, Tseng J, Nahapetyan L, Alzeerah M, Islam A. Bubbles in the box: recurrent pneumothorax from bronchopleural fistula in rheumatoid arthritis. J Investig Med High Impact Case Rep 2019; 7: 1-4.
16. Gotsman I, Goral A, Nusair S. Secondary spontaneous pneumothorax in a patient with pulmonary rheumatoid nodules during treatment with methotrexate. Rheumatology (Oxford) 2001; 40: 350-351.

17. Chen $\mathrm{CH}$, Liao WC, Liu $\mathrm{YH}$, et al. Secondary spontaneous pneumothorax: which associated conditions benefit from pigtail catheter treatment? Am J Emerg Med 2012; 30: 45-50.

18. Das SK Ghoshal B. Pneumothorax in human immunodeficiency virus infection. J Assoc Chest Physicians 2015; 3: 38-40. 